... Bertrand Kiefer: médecin, théologien, éthicien et rédacteur en chef de la «Revue Médicale Suisse»

\title{
«Réfléchir est ma passion»
}

\section{Daniel Lüthi}

Texte et illustrations

danielluethi@gmx.ch
Il est plutôt rare qu'un médecin sente l'encre d'imprimerie. C'est pourtant le cas pour Bertrand Kiefer qui fait les choses autrement que ses confrères et sa vocation d'éditeur résulte de la fusion de plusieurs métiers.

\section{La médecine - un cadavre?}

Nous parvenons à son bureau en passant par l'entrepôt de papier et l'imprimerie. Et là, nous parlons aussitôt d'un livre qui a été non seulement édité et imprimé mais aussi écrit ici: «Le cadavre de la médecine bouge encore», en l'occurrence une collection d'environ 170 chroniques rédigées ces dix dernières années par Bertrand Kiefer pour sa «Revue Médicale Suisse», constituées de réflexions approfondies sur des thèmes importants. Exactement ce qu'il faut pour commencer la nouvelle année! Empreintes de philosophie, ces chroniques contiennent beaucoup d'éléments critiques et l'ensemble dégage une note pessimiste.

La question posée au début du livre laisse entrevoir sa conclusion: «Fin de l'humanité?» Rien de moins que la fin de l'humanisme et peut-être même celle de l'humanité. «Non, je ne suis pas un pessi-

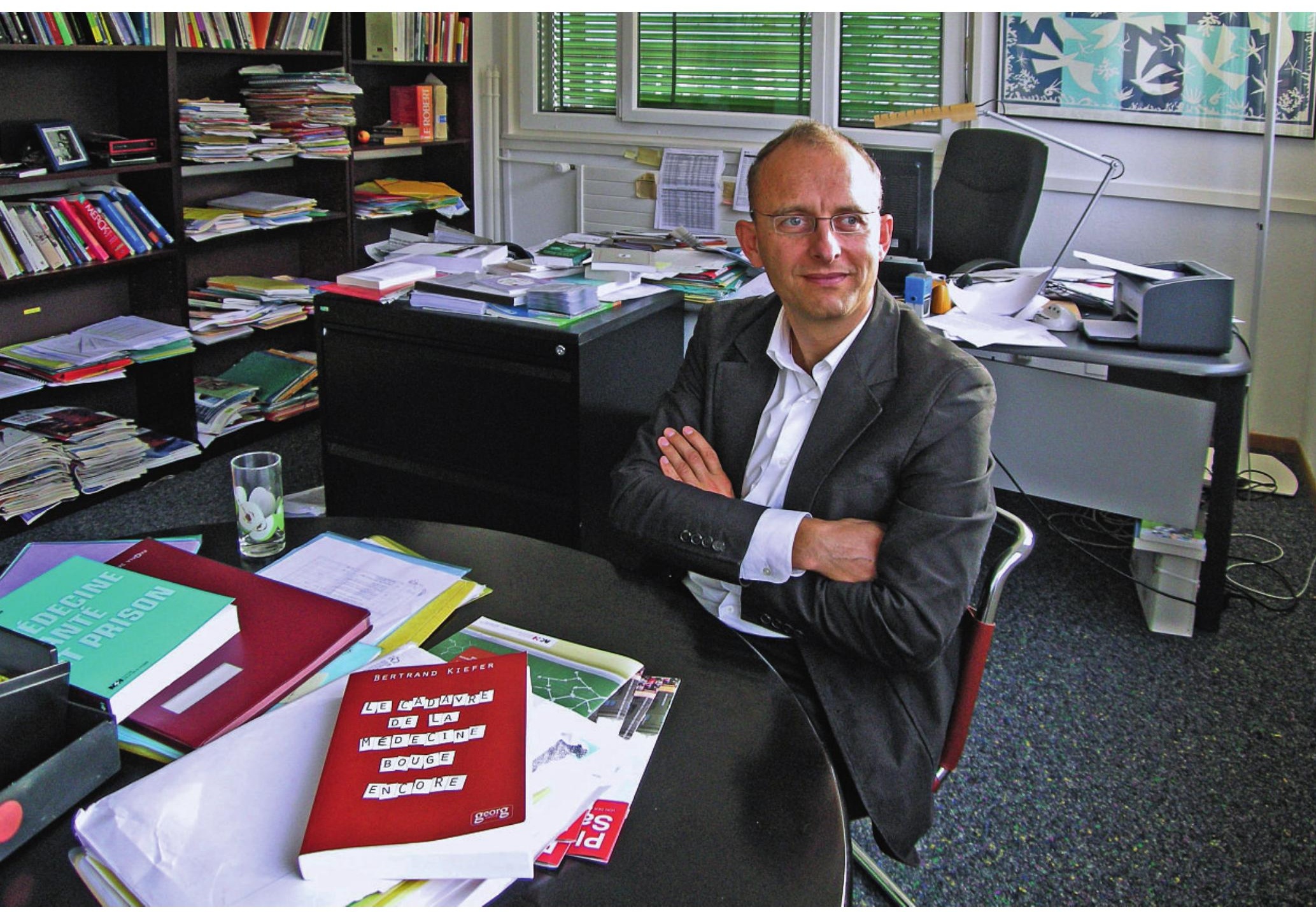


miste», se défend Bertrand Kiefer, «mais pas non plus un optimiste béat. J'essaie de voir les choses telles qu'elles sont, je suis donc plutôt un réaliste.» Concernant la médecine, il ajoute: «Toutes les cultures sont mortelles, donc la médecine peut aussi disparaître.» Il entend par là l'essence de la médecine, son humanisme, car elle se transforme graduellement en une machine à guérir, elle doit toujours plus se plier au diktat de l'économie qui empêche toute vision humanitaire. Efficacité au lieu d'empathie. Chiffres au lieu de compassion. Une médecine considérée uniquement comme un bien de consommation, un business parmi d'autres: «Les technologies, le monde virtuel et le management relèguent toujours plus l'individu au second plan», estime Bertrand Kiefer, alors qu'il s'agit pour un médecin «d'aider une personne à améliorer sa condition». Un exemple pour le comprendre: «Une vieille dame incontinente, diabétique et marchant avec difficulté: l'objectif de son médecin de famille est-il de la guérir de ses maux par tous les moyens?» Une fois de plus, Betrand Kiefer pose une question au lieu de donner une réponse, ce qui est typique pour lui.

\section{«Les technologies, le monde virtuel et le management relèguent toujours plus l'individu au second plan.»}

\section{De la prêtrise au journalisme}

Comme il le dit lui-même, Bertrand Kiefer est «un médecin qui n'a jamais exercé la médecine». Il écrit, philosophe et critique, met le doigt sur les plaies ouvertes de la société et essaie de considérer les choses avec toujours plus de distance. Gratter le vernis, rechercher, mettre au jour, découvrir, regarder derrière les coulisses: Il se sent bien comme journaliste. Mais: «Il manque malheureusement toujours plus d'argent en Suisse pour ce genre de travail et c'est un problème pour la démocratie.» On a l'impression d'entendre un politicien, ce que Bertrand Kiefer ne veut explicitement pas être. Il s'identifie plutôt aux philosophes Peter Sloterdijk, Michel Foucault, Jean Baudrillard ou Georges Canguilhem. Ce dernier a réfléchi par exemple aux limites fluctuantes entre normalité et morbidité. Bertrand Kiefer a une prédilection particulière pour ce genre de thèmes: «Réfléchir est ma passion.»

Qu'en est-il de la religion pour ce médecin et journaliste qui a été un jour prêtre catholique? On touche là un point sensible. Questionné sur les raisons qui l'ont poussé à quitter Rome et l'église, Bertrand Kiefer, apparemment gêné et restant dans l'abstrait, parle d'une situation personnelle difficile à une époque caractérisée par des dogmes et éléments doctrinaires qu'il ne voulait plus accepter et d'une femme dont il était tombé amoureux.

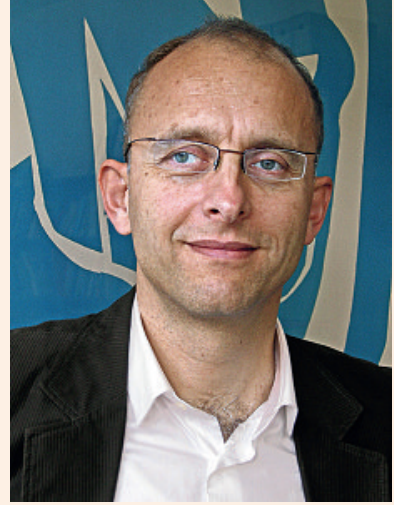

\section{Bertrand Kiefer}

Fils de médecin, Bertrand Kiefer est né en 1955

à Genève où il fréquente les écoles et fait ses études de médecine, sanctionnées en 1981 par le diplôme fédéral de médecin. Une année durant, il effectue des travaux de recherche dans le domaine des maladies infectieuses puis il se rend à Fribourg pour étudier la théologie. Consacré prêtre en 1998, il prépare un doctorat sur la technique génétique durant trois ans en séjournant en partie à Rome. Divers motifs I'incitent ensuite à quitter Rome et la prêtrise. De retour en Suisse, il travaille de 1991 à 1993 à mi-temps comme journaliste (au Journal de Genève) et à mi-temps comme psychiatre dans un service ambulatoire des HUG. En 1993, il est nommé rédacteur en chef de l'hebdomadaire «Médecine \& Hygiène» qui fusionnera en 2005 avec la «Revue Médicale de la Suisse Romande» pour former la «Revue Médicale Suisse». Depuis I'an 2000, Bertrand Kiefer est également directeur des éditions et de l'imprimerie de «Médecine \& Hygiène». Bertrand Kiefer est marié à une journaliste de la télévision. II vit avec elle et avec leur fils près de Genève. 


\section{Que nous apportera 2011?}

Tournons-nous plutôt vers la nouvelle année et jetons un regard sur 2011. «Le Managed Care et la caisse unique en seront les thèmes principaux.» Bertrand Kiefer souhaite que l'on améliore les réseaux, en particulier entre les hôpitaux et la médecine ambulatoire, et il aimerait que les caisses-maladie fassent une séparation entre le domaine social et les assurances privées.

Il ajoute: «Nous discuterons aussi des DRG, ces forfaits par cas basés sur le diagnostic qui chambouleront notre système de santé de fond en comble. Les groupes particulièrement vulnérables, par exemple les malades chroniques ou polymorbides, deviendront un problème puisque les gens doivent coûter le moins possible. Par conséquent, lorsqu'un patient dira par exemple: Je suis angoissés, le soignant devra lui répondre: 〈Moi, je n'ai pas le temps`. Les expériences faites en Allemagne nous apprennent que la pression croissante exercée sur les soignants augmente considérablement le nombre de cas d'épuisement parmi eux.» Bertrand Kiefer précise toutefois qu'il n'est pas à priori contre ce nouveau système. Une fois de plus, il plaide clairement en faveur d'un examen approfondi de la situation.

\section{Oui à l'économicité, mais pas à n'importe quel prix.}

Que lui apportera la nouvelle année? Des discussions intéressantes à la Commission nationale d'éthique concernant des questions de première importance telles que le diagnostic de préimplantation ou l'aide au suicide. Donc à nouveau des questions fondamentales concernant l'existence humaine: vivre, mourir, donner la vie ou laisser mourir.
Et sur le plan personnel? Comment envisage-t-il sa propre mort? A son habitude, Bertrand Kiefer écoute tout d'abord attentivement la question puis il réfléchit, projette son regard au loin, revient à son interlocuteur, sourit et répond: «C'est un mystère étrange. La mort est partout dans la nature. Oui, cette question me préoccupe. Autrefois, on souhaitait voir venir la mort pour pouvoir s'y préparer. Aujourd'hui, on souhaite une bonne mort plutôt soudaine, inattendue. A ce point de vue, je suis un homme moderne. J'aimerais vivre puis mourir rapidement, sans le savoir.» Vivre pleinement sa vie est pour Bertrand Kiefer par exemple: être bien accueilli par sa famille et ses amis. Avoir de nombreuses discussions enrichissantes. Ou faire des randonnées et aller skier dans les montagnes, comme il le fait chaque année en Valais durant la période des fêtes.

En 2011, Bertrand Kiefer sera naturellement aussi bien occupé par des affaires quotidiennes, dans sa fonction de directeur d'une entreprise occupant 35 personnes. Il devra faire face à des problèmes de personnel dans l'édition et l'imprimerie, aussi à la création d'un site internet important sur la santé pour la Suisse romande. «Que dois-je faire, quand...» ou «Quels sont les signes avant-coureurs d'un cas d'urgence?» telles seront les questions auxquelles il faudra répondre pour un large public. Ce projet a également besoin d'une mise en réseau étendue entre hôpitaux, sociétés cantonales de médecine ou Ligues médicales spécialisées, dit Bertrand Kiefer, qui précise: «Nous courons un risque entrepreneurial important car notre visée n'est pas d'abord commerciale.»

Bertrand Kiefer le répète: oui à l'économicité, mais pas à n'importe quel prix.

C'est donc bien vrai: les nombreux métiers de Bertrand Kiefer ont fusionné en une vocation et ce médecin, théologien, éthicien, philosophe, journaliste, rédacteur en chef et directeur d'une maison d'éditions est avant tout une personne qui aime réfléchir.

\section{La prochaine rencontre avec...»}

A la fin de chaque mois, le Bulletin des médecins suisses présente une personnalité qui s'implique dans la santé publique. En février, Daniel Lüthi parlera de sa rencontre avec Lutgard Werlen, médecin généraliste, seule médecin dans le Lötschental et alpiniste. 